\title{
ESCHER's Complex Objects: A Demonstration of Simplicity
}

\author{
B. Fischer, J. Thamm, L. Wegner, S. Wilke, and C. Zirkelbach \\ Universität Gh Kassel, FB 17, D-34109 Kassel, Germany \\ \{bufi, injt,wegner,wilke, czi\}@db.informatik.uni-kassel.de
}

\begin{abstract}
ESCHER is a visual database editor for the object-relational data model. Here, we describe the essential features of ESCHER and what is demonstrated at this conference with our prototype. Readers are invited to download the public domain software or to follow more detailed write-ups given in the references.
\end{abstract}

\section{VISUAL INFORMATION SYSTEMS}

Most data - documents, time tables, CAD drawings, etc. - have a natural, usually hierarchical structure. Visually browsing and editing these structured data has several advantages over working with "flat" relational tables; navigating on the outer levels permits travelling great distances with few key strokes, e.g. between departure cities in Figure 1, which shows parts of 260 inner-Australian flight connections from the 1995 Ansett Travel Planner. Secondly, unzooming gives a bird's view of the global structure, yet by descending into substructures, details like particular flight times can be reviewed.

When used as an editor, aggregate objects can be deleted, moved, pasted with the push of a button. Complex objects may also include multimedia types of which we currently support GIF, JPG and XBM. ESCHER may also serve as producer of HTML-code, respectively Mathematica $®$ input by means of a scripting language, called TclDB. Scripts written in TclDB, acting as methods, can be stored as ESCHER attributes as described in this proceeding's companion paper (Thamm and Wegner, 1998). 


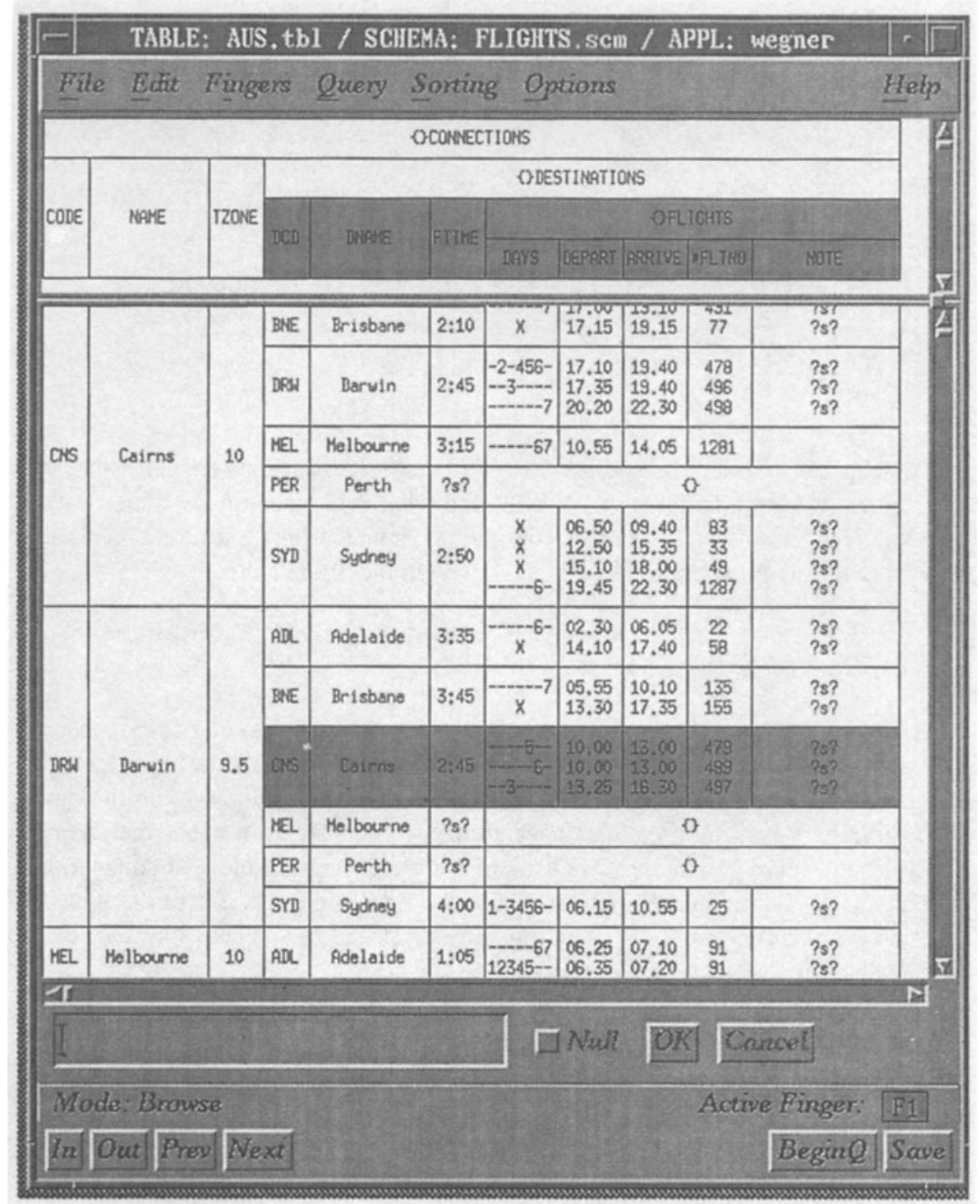

Figure 1 Navigating inside the flights database with F1 on destination Cairns

\section{FLYING OVER DATALAND}

ESCHER includes some unusual features which can best be demonstrated hands-on. At the core of ESCHER's interaction paradigm is the notion of a finger. A finger is like a cursor and points to atomic or complex objects. In Figure 1, a finger F1, 
which is depicted as a shaded area, rests on the tuple for Cairns within the set of destinations reachable from Darwin by direct flight.

There can be several fingers within one table, in a collaborative environment even fingers belonging to different users. Navigating with these fingers in and out, back and to the next item, setting them with a QBE-like query, is rather intuitive, in particular when initiated with the mouse. Figure 2 shows the control stick-like dimensional characteristics.

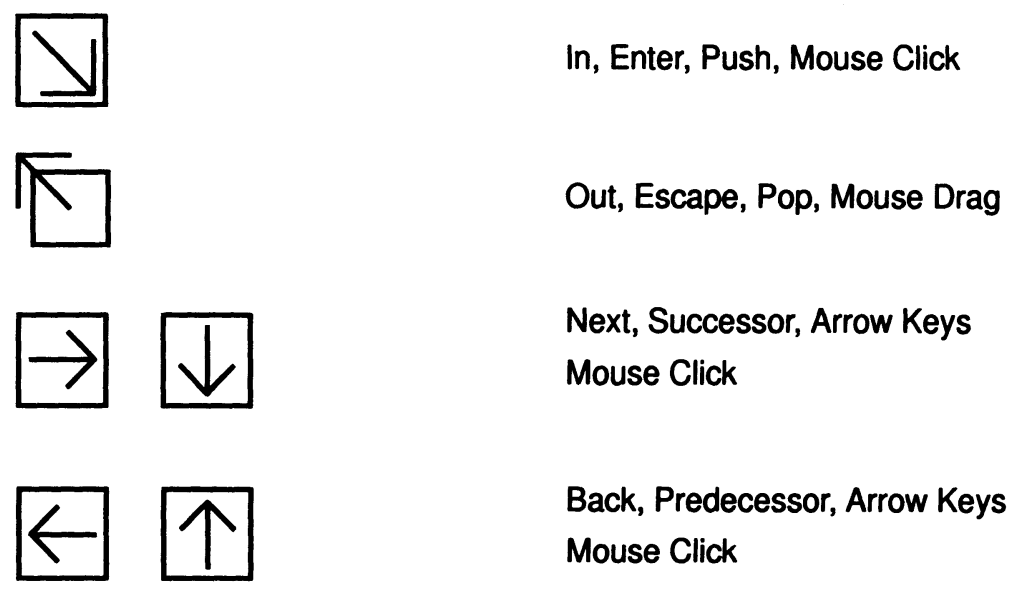

Figure 2 Basic navigational operations

Another interesting feature, which was present right from the start of the project (Wegner, 1989), is the self-referencing metaschema, which is the (infinite) schema of all schemas, including of itself, and which also has a visualization.

Other research topics treated in ESCHER are the proper handling of null values for atomic and complex objects, empty sets and lists, sorting and duplicate elimination algorithms, nesting and unnesting, efficient storage techniques - including pointer swizzling (Wegner et al., 1996) - and the use of self-referential methods for GUI and index management.

\section{OUTLOOK}

When run in a collaborative, shared environment, ESCHER follows a relaxed WYSIWIS (What You See is What I See) model and its finger paradigm gives rise to a spatial awareness concept with locality (where you placed a finger which is visible to other) and focus (the set of objects you are looking at). Although currently not supporting a multi-user environment, the demo can show the effects of following a finger, jumping to different locations, sliding a window away from a locality, etc. In particular, synchronizing navigation in this shared object space can be achieved by cheap event/operation passing as against expensive display broadcast. 
Details in connection with a tele-teaching application can be found in (Krämer and Wegner, 1998). The paper also addresses questions of different visualizations, which is not limited to nested tables, as it might appear from this demo description. However, we have not gone as far as (Haber, Ioannidis, and Livny, 1995) in permitting the user to declare new styles for schemas in any data model.

ESCHER currently runs on IBM RS6000 AIX with OSF/Motif and on LINUX. A porting to a Tcl GUI is under way. The finger paradigm has also been incorporated into the ABAP/Workbench complex object editor of SAP AG R/3 as part of a university-industry cooperation.

\section{DISCLAIMER}

ESCHER is a university prototype which support research into visualization and non-standard databases. It is in the public domain and offered "as is" with absolutely no warranty. Use for critical applications is strongly discouraged.

\section{REFERENCES}

Haber, E.M., Ioannidis, Y.E., and Livny, M. (1995) OPOSSUM: Desk-Top Schema Management through Customizable Visualization, Proc. 21st International VLDB Conference, Zurich, Switzerland (September 1995) 527-538.

Krämer, B. and Wegner, L. (1998) Beyond the Whiteboard: Synchronous Collaboration in Shared Object Spaces, IEEE Computer (in print)

Thamm, J. and Wegner, L. (1998) What You See is What You Store: DatabaseDriven Interfaces, Proc. 4th IFIP 2.6 Working Conference on Visual Database Systems (VDB4), L'Aquila, Italy, May 27-29 (eds. Yannis Ioannidis and Wolfgang Klas)

Wegner, L.(1989) ESCHER - Interactive, Visual Handling of Complex Objects in the Extended NF ${ }^{2}$-Database Model, Proc. IFIP TC-2 Working Conference on Visual Database Systems, Tokyo, Japan, April 1989, (ed. T.L.Kunii), Elsevier North-Holland Publ., Amsterdam, 277-297

Wegner L., Paul, M., Thamm, J., and Thelemann, S. (1996) Pointer Swizzling in Non-Mapped Object Stores, Seventh Australasian Database Conference (ADC'96), Melbourne, Australia, 29-30 January (ed. Rodney Topor), Australian Computer Science Communications, Vol. 18 (2) 11-20 